\title{
With hands I do not centre! Action- and object-related effects of hand-cueing in the line bisection
}

\author{
Mariagrazia Ranzini ${ }^{\mathrm{a}, *}$, Anna M. Borghi ${ }^{\mathrm{a}, \mathrm{b}, * *}$, Roberto Nicoletti $^{\mathrm{a}}$ \\ a University of Bologna, Italy \\ b ISTC-CNR, Rome, Italy
}

\section{A R T I C L E I N F O}

\section{Article history:}

Received 1 January 2011

Received in revised form 22 May 2011

Accepted 15 June 2011

Available online 26 June 2011

\section{Keywords:}

Line bisection

Action representation

Visuo-motor priming

Motor simulation

\begin{abstract}
A B S T R A C T
A variety of studies have shown action- and object-related visuo-motor priming in behavioural tasks. The peculiarity of this study lies in using a hand-cued line bisection task to explore the main properties of the motor effects evoked by action and object processing. In five experiments it is shown that flanking a line (thin vs. thick line) with images of hands (biological vs. non-biological hand) representing different actions (power vs. precision grip) biases performance towards the action more compatible with the object (power grip - thick line, precision grip - thin line). This effect is larger for the precision grip than for the power grip suggesting a functional rather than manipulative activation. In addition, the effect is larger for the biological than for the non-biological hand. We suggest that this paradigm could be potentially useful for neuropsychological studies as well as for addressing unsolved issues of embodied theories of cognition.
\end{abstract}

(c) 2011 Elsevier Ltd. All rights reserved.

\section{Introduction}

An important building block of the human ability to interact with the surrounding environment is the ability to plan and predict actions by observing objects and others interacting with objects. Neurophysiology studies on monkeys and neuroimaging studies on humans have shown that action representation is anatomically mediated by the same neural paths involved in action execution (e.g., Buccino et al., 2001; Hari et al., 1998; Grafton, 2009; Grèzes \& Decety, 2001; Rizzolatti \& Craighero, 2004; Rizzolatti et al., 1996; but see Turella, Pierno, Tubaldi, \& Castiello, 2009). Other studies have found that perceiving objects whose properties (e.g., size or orientation) are relevant for an action such as reaching or grasping, activates motor related brain areas (Chao \& Martin, 2000; CreemRegehr \& Lee, 2005; Gerlach, Law, \& Paulson, 2002; Grafton, Fadiga, Arbib, \& Rizzolatti, 1997; Johnson-Frey, 2004; Martin, 2007). The anatomical connections between action and perception systems indicate a bidirectional communication between action representation and execution (Craighero, Bello, Fadiga, \& Rizzolatti, 2002). As underlined by Grafton (2009), "What is striking [...] is that a perceptual stimulus can lead to such a largescale recruitment of the

\footnotetext{
* Corresponding author at: Dipartimento di Discipline della Comunicazione, Via Azzo Gardino 23, 40122 Bologna, Italy.

** Corresponding author at: Dipartimento di Psicologia, Viale Berti Pichat 5, 40127 Bologna, Italy.

E-mail addresses: mari.ranzini@gmail.com (M. Ranzini), anna.borghi@gmail.com, annamaria.borghi@unibo.it (A.M. Borghi).
}

brain with a complete disregard of textbook divisions between sensory and motor portions of the cortex". This quote by Grafton (2009) points to the existence of a bidirectional influence of perception on action and vice versa. Action- and object-related visuo-motor effects have been interpreted by some authors in terms of motor simulation (Decety \& Grezes, 2006; Gallese, 2009; Jeannerod, 2001; for discussion on the term see Borghi \& Cimatti, 2010). The "simulation theory [...] predicts a similarity, in neural terms, between the state where an action is simulated and the state of execution of that action" (Jeannerod, 2001). Along this line, proponents of common coding theories (Hommel, Müsseler, Aschersleben, \& Prinz, 2001; Prinz, 1997) have argued that perception- and action-related codes are functionally equivalent and represented in a common format. A number of behavioural studies have provided evidence favouring these theories through finding compatibility effects between observed or executed hands grasping postures and object size.

\subsection{Perception influences action}

The compatibility between an observed hand prime displaying either a precision or a power posture, and a target object requiring the same kind of grip, induced visuo-motor priming in several experiments (for a computational model of these effects see Caligiore, Borghi, Parisi, \& Baldassarre, 2010). What is commonly observed in these studies is that grasp observation primes the identification and categorisation of grasp-congruent target objects (e.g., Borghi et al., 2007; Vainio, Symesa, Ellis, Tucker, \& Ottoboni, 2008; see Girardi, Lindemann, \& Bekkering, 2010, for a kinematics study on contextual dependency of these effects). These effects are 
stronger when there is a high degree of overlap between the event we perceive (the observed actions) and the event we can produce through our own action: for example, kinematics evidence shows that grasp observation primes the execution of a similar action (Castiello, Lusher, Mari, Edwards, \& Humphreys, 2002; Edwards, Humphreys, \& Castiello, 2003). Behavioural evidence reveals that larger priming effects are triggered by hand primes displayed in egocentric rather than allocentric perspective (e.g., Bruzzo, Borghi, \& Ghirlanda, 2008; Vogt, Taylor, \& Hopkins, 2003), and by the observation of biological rather than robotic hands (Castiello et al., 2002; Gazzola, Rizzolatti, Wicker, \& Keysers, 2007; Pierno, Mari, Lusher, \& Castiello, 2008a; Press, Bird, Flach, \& Heyes, 2005; Tai, Scherfler, Brooks, Sawamoto, \& Castiello, 2004). Object processing also induces effects of grasp compatibility. Indeed, the objects physical characteristics were found to interact with grasp execution (e.g., size: Edwards et al., 2003; orientation: Craighero, Fadiga, Rizzolatti, \& Umilta‘, 1998; consistency: Anelli, Nicoletti, \& Borghi, 2010). However, importantly, object-related effects have also been found in the absence of a direct grasping action towards an object. For example, the kinematics of a reach-to-grasp action towards an object (movement time, deceleration time, and maximal finger aperture), performed with or without the preceding observation of the same action executed by an actor, were modulated by the presence of a second irrelevant object (Castiello, 2003). This has occurred also by means of behavioural paradigms of object categorisation where compatibility effects have been observed, either between the hand of response (left or right) and the graspable side of the object stimulus (Tucker \& Ellis, 1998), or between the kind of response (precision vs. power grip) and the evoked object grip (e.g., Ellis \& Tucker, 2000; Tucker \& Ellis, 2001, 2004).

\subsection{Action influences perception}

As perception influences action, evidence shows that action can also influence perception. Indeed, it has been demonstrated that action - both planned and observed - can bias attention towards the objects whose characteristics matched the planned or observed action. For example, object grasping facilitates the detection and discrimination of visual shapes oriented congruently with the object to grasp (Craighero, Fadiga, Rizzolatti, \& Umilta, 1999). Again, grasping preparation facilitates the detection of congruent objects, as seen in studies using selective attention paradigms (Symes, Tucker, Ellis, Vainio, \& Ottoboni, 2008; Symes, Ottoboni, Tucker, Ellis, \& Tessari, 2010). The observation of an acting hand or of a static hand that reminds the participant of a particular action can direct attention towards a compatible object even in absence of a corresponding grasping response. For example, hand posture observation that reminds the participant of a precision or power grip primed joint attention in a detection task. Fischer, Prinz, and Lotz (2008) illustrated this effect showing that participants were faster in detecting a probe appearing on one of two presented objects when the object under the probe matched the grasping posture of a previously observed hand prime. Finally, hand action observation involuntarily primed perception in a detection task with a foot response, i.e. without any involvement of hand movement (Fagioli, Hommel, \& Schubotz, 2007).

The behavioural studies described so far show that motor information is activated by the observation of objects and actions. However, several questions concerning these effects are still unclear. Firstly, it is not clear whether a static rather than a dynamic hand induces motor simulation. Neuroimaging studies have shown that human hand-object interactions, such as grasping, touching or pointing, can selectively modulate the brain activity of action observation areas (inferior frontal cortex: Johnson-Frey et al., 2003; occipitotemporal and somatosensory cortices: Pierno et al., 2008b) even in absence of a dynamic hand movement observation. Along the same line, in a TMS study by Urgesi, Moro, Candidi, and Aglioti (2006) it was found that the motor system was maximally activated during the observation of a static hand posture suggesting an implicit movement, rather than by a hand suggesting a resting position or a completed action. However, compatibility effects obtained with hand primes, even when followed by the presentation of a target object, required either a motor preparation phase (Borghi et al., 2007), or the presentation of dynamic hands (Vainio et al., 2008). Thus, it remains unclear whether observing a static effector that is not directly interacting with an object but is suggesting a grasping action, would induce a motor simulation.

Again, it is debated whether object motor information is activated automatically or is modulated by the task(e.g., Tipper, Paul, \& Hayes, 2006) and the context (e.g., Costantini et al., 2011). For example, in a recent study by Pellicano, Iani, Borghi, Rubichi, and Nicoletti (2010) it was observed that the effects induced by a torch stimulus depended both on the kind of task and on the active or passive state of the torch (i.e. switched on or off). A compatibility effect was found between the torch orientation and the response hand in a colour discrimination task (Simon-like effect) independently of the active or passive torch state. However, when participants discriminated between the upward or inverted position of the torch the compatibility effect emerged only for active state torch stimuli (functional affordance effect). Following this point, it remains unclear whether and when objects activate manipulative or functional actions (i.e. manipulate to simply grasp an object vs. manipulate to use it) (Bub, Masson, \& Cree, 2008; Borghi \& Riggio, 2009; Jax \& Buxbaum, 2010).

The present study aimed to investigate action- and objectrelated visuo-motor effects examining the aforementioned issues with a paradigm that has never been used in this context, the line bisection task. This task is an important instrument in neuropsychological diagnosis to assess brain injury syndromes associated with hemispatial deficits (e.g., Barton, Behrmann, \& Black, 1998; Bisiach, Capitani, Colombo, \& Spinnler, 1976; Kerkhoff \& Bucher, 2008; Schenkenberg \& Bradford, 1980). The line bisection task is also of great utility for research on cognition (Fischer, 2001). Indeed, this task permits the study of spatial asymmetries in healthy subjects (i.e. a consistent leftward bias, phenomenon known as "pseudoneglect": see Jewell \& McCourt, 2000). It also informs that, when lateral symbolic cues are presented at the line extremities, different kinds of bias modulation are reported demonstrating that symbolic cue processing interferes with performance (directional cues: Chieffi, 1999; Kashmere \& Kirk, 1997; other symbolic cues: De Hevia, Girelli, \& Vallar, 2006). An incredible variety of studies adopt this paradigm (see, for example, an original study by Sposito, Bolognini, Vallar, Posteraro, and Maravita (2010) on the bisection of body part to study the body representation) demonstrates that the bisection task can be flexibly and successfully applied to different contexts. For these reasons, here we conducted a series of five experiments adopting a modified version of the cued bisection paradigm, where the line stimulus was flanked by images of hands to investigate hand cueing effects.

We varied the line thickness (thin vs. thick line), the kind of hands (biological vs. non-biological hand), and the represented action (power vs. precision grip). If observing a hand near to a potentially graspable object (the line) activates motor information, then we expect a compatibility effect between hand posture and object size. In the same vein, neuroimaging studies have shown that constrained grasp action (i.e. power grasp to small objects and vice versa) modulated kinematics as well as premotor and motor brain activity, suggesting a larger difficulty to shape an unnatural rather than a natural grasping (Begliomini, Caria, Grodd, \& Castiello, 2007). In addition, if observing a hand activates functional rather than manipulation information in isolation, we predict a stronger 
effect with the precision than with the power grip. Precision and power grip have different neural representations. Indeed, neuroimaging studies have shown stronger activity of frontoparietal brain areas for skillful digit movements (i.e. non-synergistic vs. synergistic movements: Ehrsson, Kuhtz-Buschbeck, \& Forssberg, 2002; precision vs. power grasping movements: Begliomini, Wall, Smith, \& Castiello, 2007), and it has been demonstrated that the left hemisphere plays a more important role in the visual control of precision than of power grip (Gonzalez, Ganel, \& Goodale, 2006). Importantly, precision grip is more frequently associated to the functional use of objects and thus it is responsible for slower responses in reaction times because it requires more complex movements (Anelli et al., 2010; Bazzarin, Borghi, Tessari, \& Nicoletti, 2007; Borghi et al., 2007; Ehrsson et al., 2000; Vainio, Tucker, \& Ellis, 2007). Finally, if the expected effects originate from a process of simulation we should observe stronger effects with biological rather than with non-biological hands, such as a fake hand or a robotic hand, as suggested by previous studies (e.g., Press et al., 2005). For the most part, behavioural studies provide evidence for action- and object-related motor effects in humans typically seen through multiple choice tasks, where reaction times or accuracy were analysed with stimulus-response compatibility (SRC) paradigms (e.g., Ellis \& Tucker, 2000; Tucker \& Ellis, 1998). Thus, the novelty of this study also consists in the behavioural paradigm we employed, which does not require SRC.

\section{Experimental}

\subsection{Experiment 1: bisection of thin lines with biological hand flankers}

\subsubsection{Methods}

2.1.1.1. Participants. Twenty subjects (mean age: 24 , range $19-43 ; 11$ females) participated in experiment 1 . All participants declared themselves right-handed and had normal or corrected to normal vision.

2.1.1.2. Materials and procedure. The task was a computerized version of the line bisection task. Participants were required to indicate the midpoint of a line flanked by pictures of human hands (hand-line distance: $4 \mathrm{px}$ ). The left and right hands were always presented from an egocentric perspective. Participants indicated the midpoint with the help of a mouse device, whose cursor was indicated on the screen by a vertical arrow (in white with a black border, size: $17 \times 7 \mathrm{px}$ ) under the line (see Fig. 1). The arrow cursor was fixed on the vertical axis, so that participants could move it only through the horizontal axis. Each stimulus was displayed on the screen until response. The line was centrally presented on the horizontal axis, but its vertical position (up or down from the screen centre) and the arrow-cursor initial position (left or right) were randomly assigned to each trial. The line was a thin line (4 px height) varying in length (short line: 288 px; long line: 432 px). Each hand-flanker represented a power or a precision grip (stimuli measurement details are reported in Table 1$)$. The hand-flanker pairs belonged to four conditions: power left + power right (POPO); power left + precision right (POPR); precision left + power right (PRPO); precision left + precision right (PRPR). There were 8 practice trials and 80 experimental trials ( 10 for each LineLength $(2) \times$ GripPosition (4)).

Participants were required to indicate for each trial the midpoint of the line with the help of the mouse device (Fig. 1). To emphasize the presence of hand flankers and the stimuli features, as well as the flankers irrelevance for the execution of the task, instruction given were as follows:

"You will see each time a black line flanked by images of hands. Your task is to shift the arrow cursor towards the midpoint with the help of the mouse device, and to press the left button. Note that the flankers are completely irrelevant of the execution of the task, so that you have to judge the midpoint of the length occupied by the black line only. Please try to be as accurate as possible avoiding to spend too much time to respond to each trial".

Participants were instructed to keep a central position at a distance of about $55 \mathrm{~cm}$ from the screen (screen resolution: $1280 \times 1024)$. The experiment lasted about 6-8 minutes, and at the end participants were informed about the experimental aims.

2.1.1.3. Data analysis and results. For each trial, we analysed the accuracy expressed in pixels (i.e. subjective minus real midpoint: so a positive or a negative value corresponded respectively to a rightward or leftward bias). Outliers from the accuracy (2.5 SD cut-off for each subject and line length) were excluded from analyses ( $1 \%$ of trials eliminated). Statistical analyses were conducted by means of ANOVAs (using the correction of Greenhouse-Geisser), and then $t$-tests for post hoc analyses (using the correction of Bonferroni on main effects).

Accuracy was firstly statistically analysed using repeated measures ANOVAs with LineLength (long, short), and GripPosition (POPO; POPR; PRPO; PRPR) as within-subject factors. LineLength was significant $(F(1,19)=5.033, p<0.05)$, indicating a larger leftward bias for long (mean $=-2.84, \mathrm{SEM}=0.83$ ) than short lines ( mean $=-1.51$, SEM $=0.48)$. GripPosition was also significant $(F(3,17)=50.300$ $p<0.001$ ), indicating that in the condition of grip sameness (same grip on left and right) the leftward bias was reduced with the precision grip (POPO: mean $=-2.85$, $\mathrm{SEM}=0.59$; PRPR: mean $=-1.36, \mathrm{SEM}=0.70$, Fig. $2 \mathrm{a}$ ), whereas in the condition of grip diversity (i.e. different grip on left and right) subjects misplaced the midpoint towards the precision grip (POPR: mean $=2.58, \mathrm{SEM}=0.90$; PRPO: mean $=-7.06$ $\mathrm{SEM}=0.90$, Fig. $2 \mathrm{~b})$. The interaction was significant $(F(3,17)=35.608, p<0.001)$, indicating a modulation of the GripPosition effect in relation to the line length (Table 2 ). All comparisons on GripPosition were significant (all $p s<0.05$; but POPO vs. PRPR: $p=0.06$ ). All comparisons on GripPosition for each line length were significant (all $p s<0.05$ ), except POPO versus PRPR (long line: $p>0.1$; short line: $p=0.06$ ), and POPR versus PRPR with short lines $(p=0.06)$.

2.1.1.4. Summary of results. Experiment 1 revealed effects of hand-cueing in the line bisection task. Indeed, in conditions of grip diversity task-irrelevant hands placed at the extremities of the line induced a misplacement of the midpoint towards the precision grip posture, whereas in conditions of grip sameness a marginally significant rightward bias for precision grip was observed. This last pattern of results suggests that the most relevant hand (i.e. the right hand: dominant or in use) is associated with the precision grip action.

\subsection{Experiment 2: bisection of thick lines with biological hand flankers}

\subsubsection{Methods}

2.2.1.1. Participants. Twenty subjects (mean age: 27 , range $18-44 ; 10$ females) participated in experiment 2 . All participants declared themselves right-handed and had normal or corrected to normal vision.

2.2.1.2. Materials and procedure. Materials and procedure were identical to experiment 1 except for the fact that the line stimulus was here a thick line (68 px height).

2.2.1.3. Data analysis and results. Scoring and analysis were identical to experiment 1 ( $1 \%$ of outlier responses were eliminated).

For experiment 2, LineLength was significant $(F(1,19)=13.596, p<0.01)$ indicating a leftward bias for long (mean $=-1.49, \mathrm{SEM}=0.93$ ) but not for short lines (mean $=0.10, \mathrm{SEM}=0.81$ ), not significantly interacting with GripPosition (Table 2). The main effect of GripPosition was significant $(F(3,17)=4.416, p<0.05)$. However, in this experiment conditions of grip diversity shifted the midpoint towards the power grip (POPR: mean $=-1.46, \mathrm{SEM}=0.98$; $\mathrm{PRPO}$ : mean $=-0.01, \mathrm{SEM}=0.76$ Fig. 2b), whereas with the same flankers grip there was a reduced leftwards shift for the power grip (POPO: mean $=-0.51, \mathrm{SEM}=0.95$; $\mathrm{PRPR}$ : mean $=-0.79, \mathrm{SEM}=0.82$, Fig. 2a). All comparisons on GripPosition were not significant (all $p s>0.1$ ), except POPR versus PRPO $(t(19)=-3.049 p<0.05)$ and POPO versus POPR $(p=0.05)$.

2.2.1.4. Comparison between experiments 1 and 2. Experiments 1 and 2 were compared separately for the conditions of grip sameness and diversity. The ANOVA with GripSameness (POPO; PRPR) as a within-subject factor and LineThickness (thin: experiment 1 ; thick: experiment 2 ) as between-subjects factor revealed a marginally significant effect of GripSameness $(F(1,38)=3.617, p=0.07)$ indicating a larger leftward bias in POPO (mean $=-1.68)$ than PRPR $($ mean $=-1.07)$. LineThickness was not significant $(p>0.1)$. The factors interaction was significant $(F(1,38)=7.851, p<0.01)$, indicating a larger leftward bias with thin than with thick lines in the power grip $(t(38)=-2.107 ; p<0.05)$ but not in the precision grip condition. The ANOVA using GripDiversity (POPR; PRPO) as the within-subject factor and LineThickness (thin; thick) as the between-subjects factor revealed a significant effect of GripDiversity $(F(1,38)=38.785, p<0.001)$ indicating a leftward bias for PRPO (mean $=-3.54$ ) but not for POPR (mean $=0.56$ ). LineThickness was not significant $(p>0.1)$. The interaction was significant $(F(1,38)=71.081, p<0.001$, and both comparisons were significant (thin vs. thick in POPR: $t(38)=3.041, p<0.005$; in PRPO: $t(38)=-6.015$, $p<0.001)$.

We then compared by means of $t$-tests the indexes of the effects of GripPosition across experiments. To perform these comparisons, we calculated the indexes of the effects for grip sameness (Fig. 2a) and grip diversity (Fig. 2b) for each of the experiments. For each subject we subtracted the condition with the most positive (or least negative) mean value from the condition with the more negative mean value. In this way, a positive value corresponded to a bias in the observed direction for each experiment. We observed that the effects of precision grip in experiment 1 were larger than the effects of power grip in experiment 2 (grip sameness: $t(38)=1.902$ $p=0.07$; grip diversity: $t(38)=6.228, p<0.001$, Fig. $2 \mathrm{a}$ and $\mathrm{b}$ ).

2.2.1.5. Summary of results. Experiment 2 confirmed the hypothesis that handcueing generate motor-related effects, both specific for the object (the line), and non-specific effects of grip. Indeed, with a thick line in conditions of grip diversity 


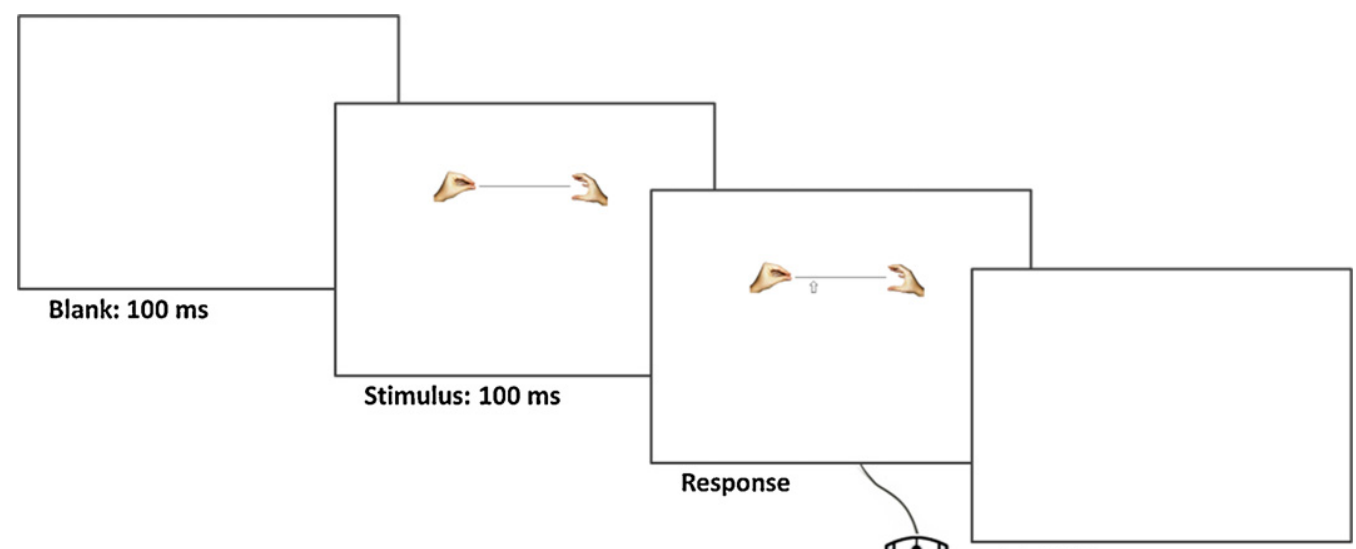

ITI: $1000 \mathrm{~ms}$

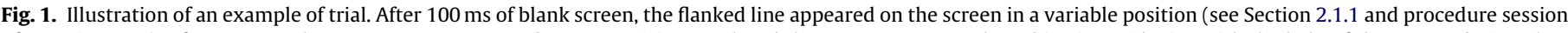

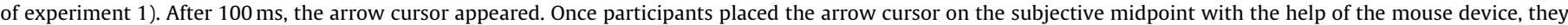

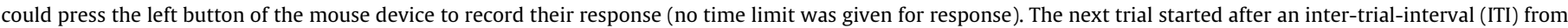
the response onset of $1000 \mathrm{~ms}$ (blank screen).

Table 1

Stimuli measurement details (maximal length and height) of each stimulus expressed in pixel.

\begin{tabular}{|c|c|c|c|}
\hline & Stimulus & Image length (px) & Image height (px) \\
\hline \multirow[t]{2}{*}{ Experiments 1-2 } & Power bio hand & 168 & 150 \\
\hline & Precision bio hand & 193 & 140 \\
\hline \multirow[t]{2}{*}{ Experiment 3} & Power fake hand & 167 & 150 \\
\hline & Precision fake hand & 207 & 150 \\
\hline \multirow[t]{4}{*}{ Experiments 4-5 } & Power bio hand & 168 & 150 \\
\hline & Precision bio hand & 168 & 126 \\
\hline & Power robotic hand & 168 & 150 \\
\hline & Precision robotic hand & 168 & 114 \\
\hline
\end{tabular}

More information concerning the stimuli features can be found at the following link: http://laral.istc.cnr.it/borghi/Ranzinietal_BisectionGrip_Table1.pdf.

task-irrelevant hands placed at the extremities of the line induced a misplacement of the midpoint towards the power grip, i.e. with the grip more compatible with the line thickness. In conditions of grip sameness, instead, non-specific effects of grip indicated a marginally significant rightward bias for the power grip posture. Interestingly, the effects of the precision grip with biological hands in experiment 1 were larger than the effects of the power grip in experiment 2 . This is in line with the hypothesis that predicted a predominant activation of functional rather than manipulative object motor information. In particular, the comparison of experiments 1-2 in conditions of grip sameness revealed - although marginally significant - an effect of grip. It is important to notice that this last pattern of results is compatible with a

Table 2

Line bisection performance (mean bisection error and SEM) for each condition of experiments 1-3.

\begin{tabular}{|c|c|c|c|c|c|c|c|}
\hline \multirow[t]{2}{*}{ LineLength } & \multirow[t]{2}{*}{ GripPosition } & \multicolumn{2}{|c|}{ Experiment 1} & \multicolumn{2}{|c|}{ Experiment 2} & \multicolumn{2}{|c|}{ Experiment 3} \\
\hline & & Mean & SEM & Mean & SEM & Mean & SEM \\
\hline Long & POPO & -3.550 & 0.833 & -1.255 & 1.009 & -5.080 & 1.338 \\
\hline Long & POPR & 3.956 & 1.121 & -2.481 & 1.109 & -1.466 & 1.346 \\
\hline Long & PRPR & -2.100 & 0.998 & -1.546 & 0.943 & -3.685 & 1.332 \\
\hline Long & PRPO & -9.654 & 1.233 & -0.659 & 0.946 & -7.617 & 1.376 \\
\hline Short & POPO & -2.149 & 0.436 & 0.244 & 0.978 & -2.625 & 0.856 \\
\hline Short & POPR & 1.197 & 0.758 & -0.442 & 0.918 & -0.823 & 0.893 \\
\hline Short & PRPR & -0.610 & 0.560 & -0.037 & 0.750 & -2.513 & 0.830 \\
\hline Short & PRPO & -4.475 & 0.687 & 0.635 & 0.773 & -4.621 & 0.813 \\
\hline
\end{tabular}

Table 3

Line bisection performance (mean bisection error and SEM) for each condition of experiments 4-5.

\begin{tabular}{|c|c|c|c|c|c|c|}
\hline \multirow[t]{2}{*}{ HandType } & \multirow[t]{2}{*}{ LineThickness } & \multirow[t]{2}{*}{ GripPosition } & \multicolumn{2}{|c|}{ Experiment 4} & \multicolumn{2}{|c|}{ Experiment 5} \\
\hline & & & Mean & SEM & Mean & SEM \\
\hline Bio & Thin & POPR & -0.806 & 0.769 & -4.486 & 0.795 \\
\hline Bio & Thin & PRPO & -9.901 & 0.881 & -7.146 & 0.638 \\
\hline Bio & Thick & POPR & -5.543 & 1.183 & -4.671 & 0.982 \\
\hline Bio & Thick & PRPO & -2.439 & 1.336 & -4.436 & 1.141 \\
\hline Robotic & Thin & POPR & -4.000 & 0.736 & -5.161 & 0.628 \\
\hline Robotic & Thin & PRPO & -7.570 & 0.817 & -5.104 & 0.631 \\
\hline Robotic & Thick & POPR & -5.243 & 1.137 & -5.029 & 0.855 \\
\hline Robotic & Thick & PRPO & -4.315 & 1.185 & -5.050 & 1.024 \\
\hline
\end{tabular}


a

\section{(Precision - Precision)}
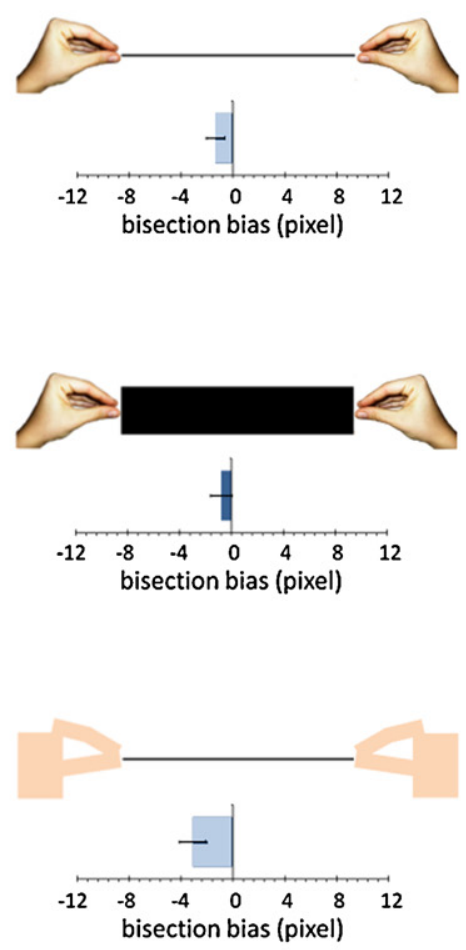

b

PRPO

(Precision - Power)
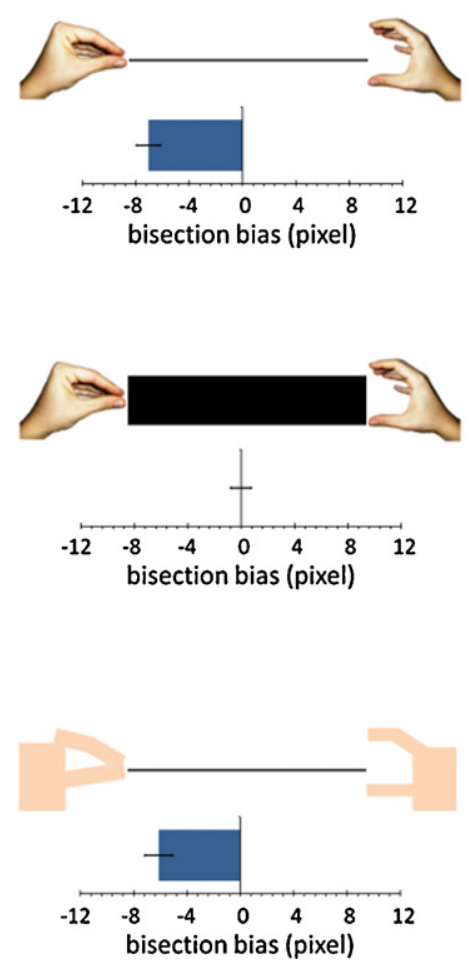

POPO

(Power - Power)
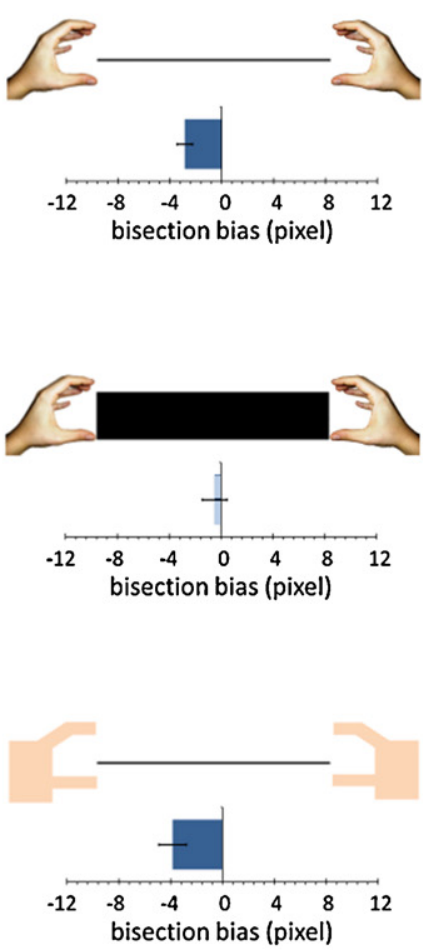

POPR

(Power - Precision)
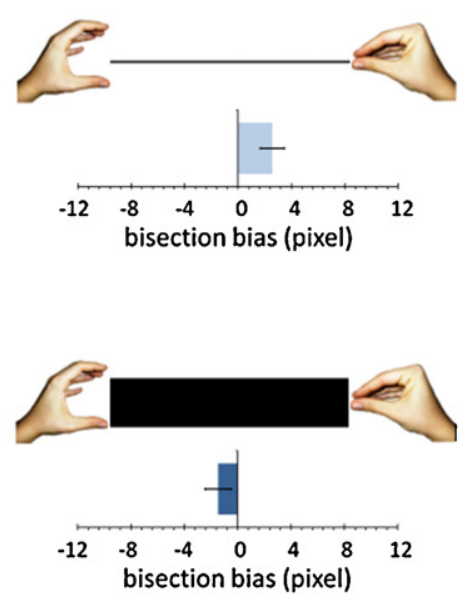

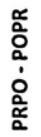

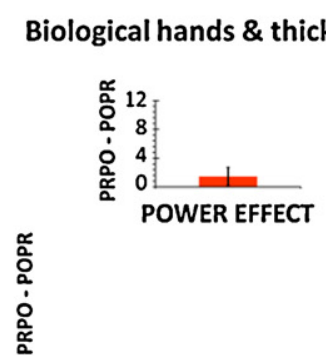

Fake hands $\&$ thin line

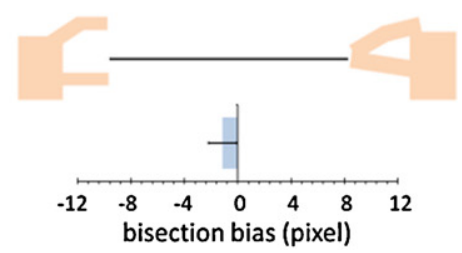

Biological hands \& thin line

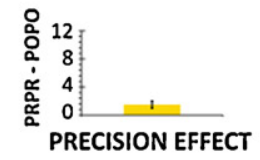

Biological hands \& thick line

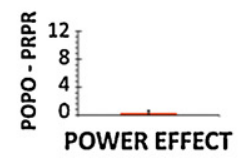

Fake hands \& thin line

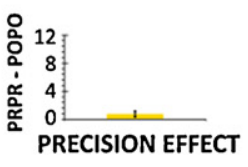

\section{GRIP EFFECTS}

Biological hands \& thin line

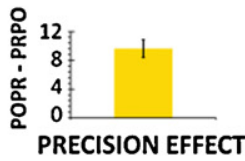

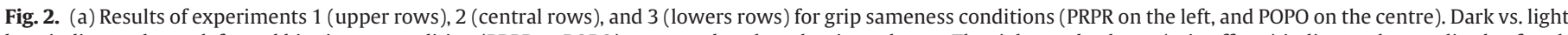

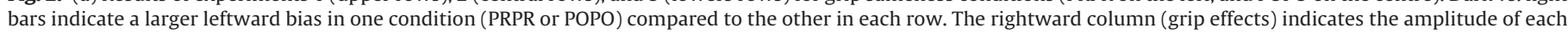

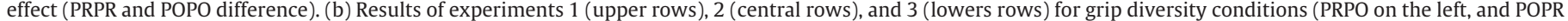

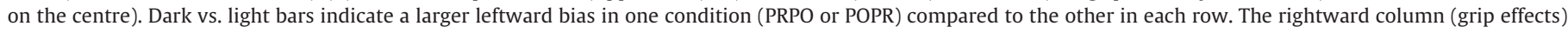
indicates the amplitude of each effect (PRPO and POPR difference). 
motor simulation interpretation because it indicates that the most relevant action is more strongly associated with the most relevant hand (i.e. the right hand: dominant or in use) for a potential execution of that action.

\subsection{Experiment 3: bisection of thin lines with pincer-like flankers}

Experiment 3 was carried out originally to exclude the possibility that lowlevel mechanisms (i.e. physical properties of the stimuli) could be responsible for the observed effects: for this reason, biological hands of experiments 1 and 2 were replaced by pincer-like images created ad hoc for each hand stimulus.

\subsubsection{Methods}

2.3.1.1. Participants. Twenty subjects (mean age: 20 , range $19-34 ; 15$ females) participated in experiment 3. All participants declared themselves right-handed and had normal or corrected to normal vision.

2.3.1.2. Materials and procedure. Methods and procedure were identical to experiment 1 except for the fact that the space occupied by the hands in experiment 1 was replaced by an ensemble of pink rectangles (stimuli measurement details are reported in Table 1). In the instructions they were called geometric images, and not hands nor pincer-like objects. The reason for this was to prevent the association of the stimuli to hands or to pincer-like objects, in order to check simply for low-level perceptual mechanisms. At the end of experiment 3 subjects were asked what was similar to the images they observed.

2.3.1.3. Data analysis and results. Scoring and analysis were identical to experiment 1 ( $2 \%$ of outlier responses were eliminated).

Even if participants were not instructed to consider stimuli as pincer-like objects or as fake-hands, at the end of the experiment only five participants reported to have perceived the geometric images as something other than fake hands ( 2 missing). For this reason, we will refer hereafter to these flankers as fake hands. In our opinion, this means that the effects here observed were plausibly originated by cognitive mechanisms which were similar to those of experiment 1 . Effects of LineLength $(F(1,19)=6.682, p<0.05)$ and GripPosition $(F(3,17)=26.980, p<0.001)$ were significant, as well as their interaction $(F(3,17)=4.079, p<0.05$, Table 2$)$. Indeed, a larger leftward bias was observed for long (mean $=-4.46, \mathrm{SEM}=1.26$ ) than for short lines (mean $=-2.65, \mathrm{SEM}=0.77$ ). Again, the main effect of GripPosition indicated that in the condition focusing on grip diversity subjects misplaced the midpoint towards the precision grip (POPR: mean $=-1.14$, SEM $=1.05$; PRPO: mean $=-6.12$, $\mathrm{SEM}=1.03$, Fig. $2 \mathrm{~b}$ ), whereas in the conditions of grip sameness a minor difference was observed (POPO: mean $=-3.85, \mathrm{SEM}=1.06$; PRPR: mean $=-3.099, \mathrm{SEM}=1.03$, Fig. 2a). All comparisons of GripPosition were significant (all $p s<0.05$ ), except for POPO versus PRPR $(p<0.1)$. All comparisons of GripPosition in each line length were significant (all $p s<0.05$ ), except for POPO versus PRPR both with the short and long lines, POPO versus PRPO with short lines, and POPR versus PRPR with long lines (all ps $>0.1$ ).

2.3.1.4. Comparison between experiments 1 and 3. Experiments 1 and 3 were compared separately for the conditions of grip sameness and diversity. The ANOVA with GripSameness (POPO; PRPR) as the within-subject factor and HandType (bio; fake) as the between-subjects factor revealed an effect of GripSamenes $(F(1,38)=11.622, p<0.005)$ indicating a larger leftward bias for POPO (mean $=-3.35)$ than PRPR (mean $=-2.23$ ). HandType was not significant, nor was the interaction. The ANOVA with GripDiversity (POPR; PRPO) as the within-subject factor and HandType (bio; fake) as the between-subjects factor revealed an effect of GripDiversity $(F(1,38)=99.569, p=0.001)$ indicating a leftward bias for PRPO (mean $=-6.59)$ but not for POPR (mean $=0.72)$. HandType was not significant $(p>0.1)$. The significant factors interaction $(F(1,38)=10.148, p<0.005)$ indicated that POPR was the only factor to induce a significantly larger rightward bias with biological than fake hands $(t(38)=2.695 ; p<0.02)$.

To compare the effects of GripPosition across experiments, we calculated the indexes of the effects for grip sameness (Fig. 2a) and grip diversity (Fig. 2b) for each of the experiments and we compared them by means of $t$-tests. For each subject we subtracted the condition with the most positive (or least negative) mean value from the condition with the more negative mean value. In this way, a positive value corresponded to a bias in the observed direction for each experiment. Precision and power grip effects did not differ in the conditions of grip sameness $(p>0.1)$, but in conditions of grip diversity the effect of the precision grip was significantly larger in experiment 1 than in experiment $3(t(38)=3.186, p<0.01$, Fig. $2 \mathrm{a}$ and $\mathrm{b})$.

2.3.1.5. Summary of results. Experiment 3 suggests that the effect of hand-cueing observed in experiments 1 cannot be attributed only to low-level perceptual effects of asymmetries between stimuli, in that it was significantly smaller with fake than with biological hands, at least in conditions of GripDiversity.
2.4. Experiments 4 and 5: bisection of thin or thick lines with biological or robotic hand flankers

To strengthen our conclusions, we replicated and extended the results through the design of experiments 4 and 5. We collapsed all the relevant factors (line thickness, kind of hand, and action position) in a within-subjects design. In experiment 5 a vertical line dividing the line from the hand was added. We hypothesized a cancellation of the effects when the relation between the object and the stimulus is hindered by the vertical line.

\subsubsection{Methods}

2.4.1.1. Participants. Fourteen subjects (mean age: 21 , range 19-33; 10 females) participated in experiments 4 and 5 ( 8 subjects began with experiment 4 , and 6 subjects started with the experiment 5 ). All participants declared themselves righthanded and had normal or corrected to normal vision.

2.4.1.2. Materials and procedure. Line length slightly differed (392 or $432 \mathrm{px}$ ), so this factor was not considered for analyses. The hand-flankers represented biological or robotic hands, indicating either power or precision grip actions (stimuli measurement details are reported in Table 1 ). The hand position had two levels (POPR or PRPO). There were 16 training trials and 80 experimental trials ( 10 for each LineThickness $(2) \times$ HandType (2) $\times$ HandPosition (2) condition). In experiment 5 a vertical black line $(4 \mathrm{px} \times 150 \mathrm{px})$ was presented at each line extremity.

2.4.1.3. Data analysis and results. Outliers from the accuracy (2.5 SD cut-off for each subject) were excluded from analyses ( $2 \%$ for experiment $4 ; 1 \%$ for experiment 5 ). Accuracy was firstly statistically analysed with a repeated measure ANOVA with HandType (biological, robotic), LineThickness (thin, thick) and GripPosition (POPR, PRPO) as within subject factors, and $t$-tests were used of post hoc analyses. A preliminary ANOVA including Experiment as a fourth factor resulted in a significant interaction between all factors $(F(1,13)=5.762, p<0.05)$. Analyses for each experiment are reported here.

Experiment 4 revealed a main effect of HandType $(F(1,13)=5.294, p<0.05)$ indicating a larger leftward bias for the robotic (mean $=-5.282, \mathrm{SEM}=0.80$ ) than the biological hand ( $m e a n=-4.672$, SEM $=0.86$ ). GripPosition was also significant $(F(1,13)=28.644, p<0.001)$ indicating a larger leftward bias for PRPO (mean $=-6.056, \mathrm{SEM}=0.89)$ than POPR $($ mean $=-3.898, \mathrm{SEM}=0.80)$. GripPosition interacted with HandType $(F(1,13)=10.419, p<0.01)$, and with LineThickness $(F(1,13)=91.093, p<0.001)$. In particular, the difference between PRPO and POPR was larger for the biological (PRPO: mean $=-6.170, \mathrm{SEM}=0.96$; POPR: mean $=-3.175, \mathrm{SEM}=0.82$ ) than the robotic hand $(\mathrm{PRPO}$ : mean $=-5.943$, $\mathrm{SEM}=0.87$; POPR: mean $=-4.621$, $\mathrm{SEM}=0.81$ ). Again, the difference between PRPO and POPR was larger for thin (PRPO: mean $=-8.735, \mathrm{SEM}=0.80$; POPR: mean $=-2.403, \mathrm{SEM}=0.69$ ) than thick lines (PRPO: mean $=-3.377, \mathrm{SEM}=1.22$; POPR: mean $=-5393, \mathrm{SEM}=1.14)$. The triple interaction between factors was also significant $(F(1,13)=27.410, p<0.001$, Table 3 and Fig. 3$)$. No other main effects or interactions were significant. Taken together, this pattern of interactions suggests that the grip effect was modulated by the kind of object (thin or thick line) and hand (biological or robotic), thus confirming previous experiments. Post hoc comparisons were conducted to explore the nature of these interactions: the comparisons between POPR and PRPO in each HandType $\times$ LineThickness condition were all significant (all $p s<0.01$ ), except in the robotic hand - thick line condition $(p>0.1)$. Both precision and power grip effects (Fig. 3) were significantly larger $(t(13)=5.414, p<0.001 ; t(13)=2.861, p<0.05$, respectively) with the biological (precision effect: mean $=9.09, \mathrm{SEM}=0.76$; power effect: mean $=3.10, \mathrm{SEM}=0.66$ ) than the robotic hand (precision effect: mean $=3.57$, $\mathrm{SEM}=0.93$; power effect: mean $=0.93, \mathrm{SEM}=0.59$ ) (Fig. 3 ). Again, the precision grip effect was significantly larger than the power grip effect both in the biological $(t(13)=6.458, p<0.001)$ and in the robotic hand $(t(13)=2.671, p<0.05)$ conditions.

In experiment 5 , as predicted, no significant main effects or interactions were found. Only the HandType $\times$ LineThickness was marginally significant $(F(1,13)=3.548, p=0.08)$, as well as the triple interaction between all factors $(F(1,13)=4.570, p=0.05$, Table 3 and Fig. 4$)$. Post hoc comparisons on GripPosition in each LineThickness and HandType revealed no significant differences in POPR versus PRPO, except in the thin lines - biological hand condition $(t(13)=2.906, p<0.05)$. Precision and power grip effects (Fig. 4) did not differ, neither in the biological (precision effect: mean $=2.66$, $\mathrm{SEM}=0.92$; power effect: mean $=0.24, \mathrm{SEM}=0.88$ ) nor in the robotic hand condition (precision effect: mean $=-0.05, S E M=0.78$; power effect: mean $=-0.02, \mathrm{SEM}=0.76$ ). However, the effect of precision was significantly larger for the biological hand condition compared to the robotic one $(t(13)=2.948$, $p<0.05)$.

2.4.1.4. Comparison between experiments 4 and 5. The indexes for the precision grip effect and for the power grip effect for each HandType were also calculated (precision grip effect: POPR minus PRPO for thin lines; power grip effect: PRPO minus POPR for thick lines). In this way, a positive value corresponded to a bias in the observed direction, whereas a negative value corresponded to a bias in the opposite direction (Figs. 3 and 4 ). The comparisons between experiment 4 and 5 , performed by means of 
PRPO

(Precision - Power)
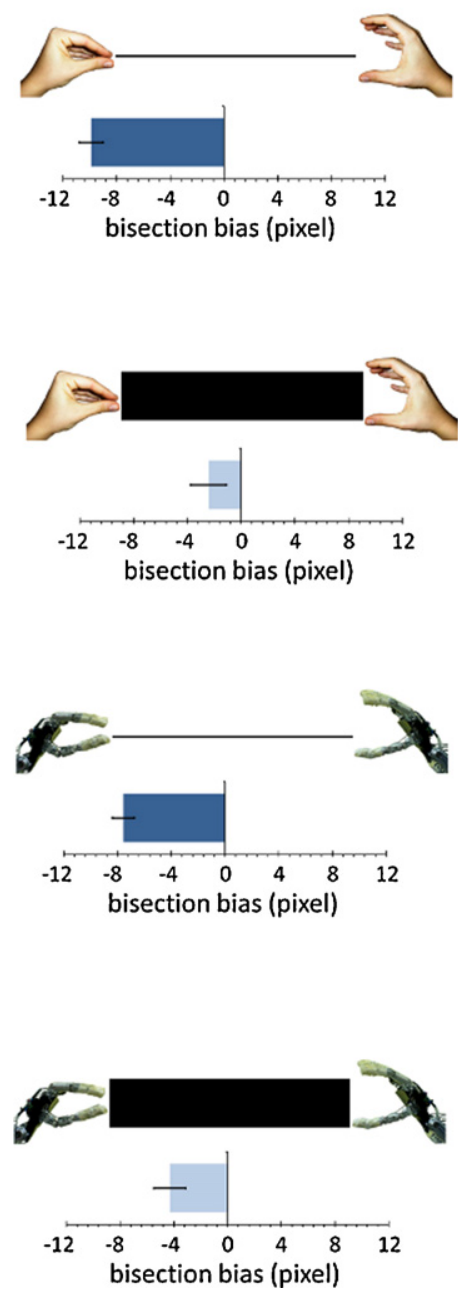

POPR

(Power - Precision)
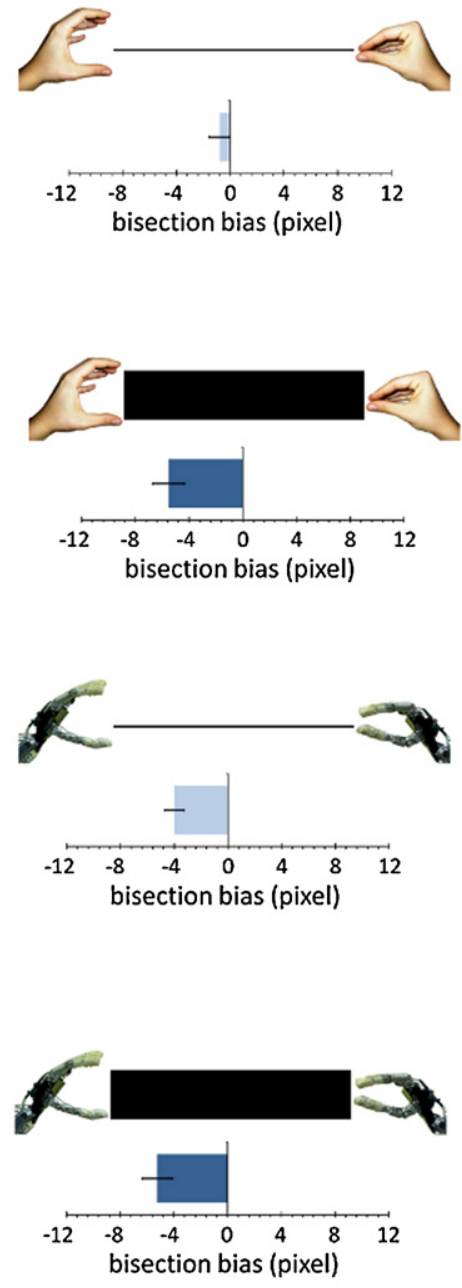

GRIP EFFECTS

Biological hands \& thin line

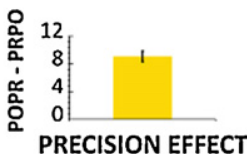

Biological hands \& thick line

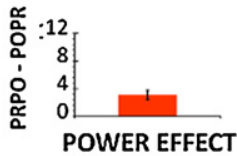

Robotic hands \& thin line

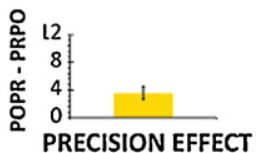

Robotic hands \& thick line

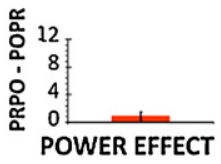

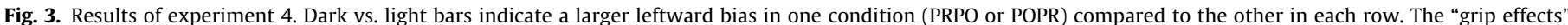

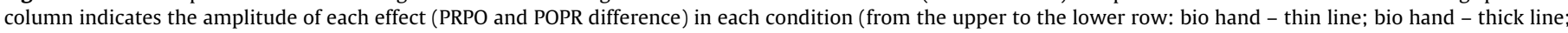
robotic hand - thin line; robotic hand - thick line).

$t$-tests, for each precision or power grip effect $\mathrm{x}$ biological or robotic hand conditions were all significant (all $p s<0.05$ ), except for the robotic hand - thick line condition $(p>0.1)$. Experiments did not significantly differ in the overall performance (exp. 4: mean $=-4.98, \mathrm{SEM}=0.82$; exp. 5 : mean $=-5.14, \mathrm{SEM}=0.67, p>0.8$ ).

2.4.1.5. Summary of results. Experiments 4 and 5 replicated and extended previously observed results. In experiment 4 , the performance was modulated by the compatibility between the kind of grip represented by the hand and the kind of line-target: with the thin line subjects misperceived the midpoint towards the hand representing a precision grip, and with the thick line subjects performed according to the opposite bias. Crucially, the precision grip effect was larger both for the biological and the robotic hands conditions, as observed in previous experiments. This result again suggests functional rather than manipulative object motor information. In addition, both the precision and the power grip effects were significantly smaller in the robotic hand condition than in the biological one, in line with the simulation hypothesis. Finally, experiment 5 revealed that when the relation between the action and target was prevented by a vertical black line, the effects of grip mostly disappeared, indicating that perceptual factors in isolation cannot be responsible for the observed effects.

\section{General discussion}

We made use of a hand-cued line bisection task to investigate action- and object-related visuo-motor cueing effects. The general idea was to examine, with a novel paradigm, the effects of grasp- ing compatibility between the hand posture and the line target, as a demonstration that action processing is triggered even with task-irrelevant hands cueing in a paradigm not involving SRC. We modulated line thickness (thin, thick), kind of hand (biological, non-biological), and kind of hand-cues grip (precision vs. power) in condition of grip sameness (same grip on the left and right: POPO, PRPR) or diversity (different grip on the left and right: POPR, PRPO). Overall, our results confirmed the advanced predictions. They revealed that observing a biological hand in potential interaction with a special kind of object, i.e. a line, activates a motor simulation, even if the hand posture is not relevant to the task. Even without using a SRC paradigm, we found a compatibility effect between the hand posture (precision, power) and the line (thin, thick). This suggests that attention is directed where the hand posture is congruent with the line width (see Fischer et al., 2008), i.e. where an action opportunity is present. The stronger lateralization effect obtained with the precision than with the power hand posture allows us to conclude not only that a motor simulation of object grasping occurs, but that this motor simulation is rather fine-grained and detailed, as with the precision grip a skilled, functional action is simulated. Finally, the stronger effect found with biological than with non biological hands strongly suggests that a 
PRPO

(Precision - Power)
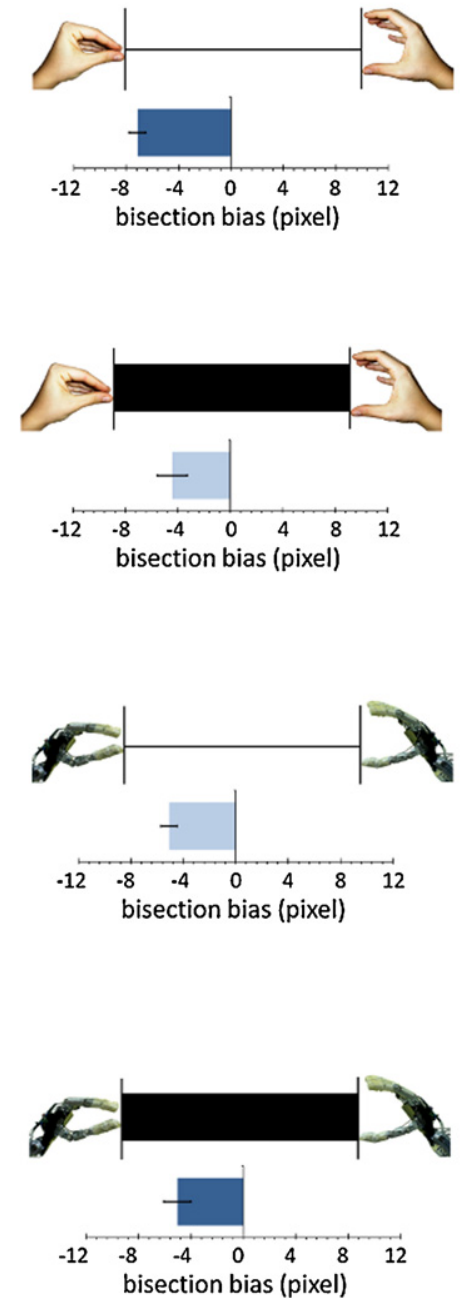

POPR (Power - Precision)
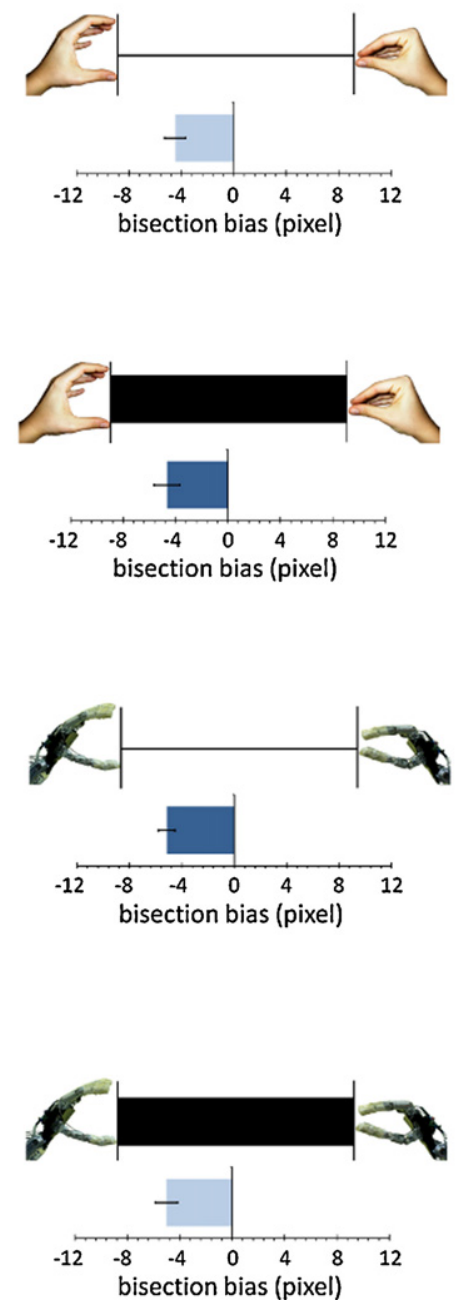

GRIP EFFECTS

Biological hands \& thin line

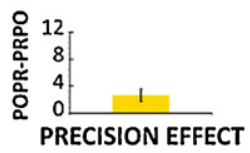

Biological hands \& thick line

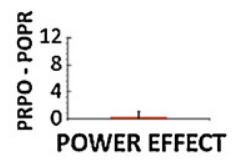

Robotic hands \& thin line

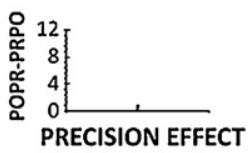

Robotic hands \& thick line

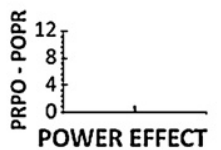

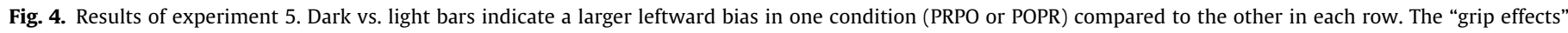

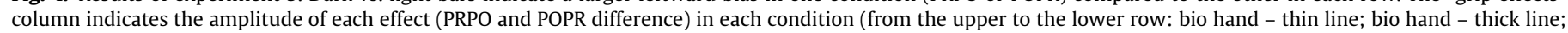
robotic hand - thin line; robotic hand - thick line).

mirroring mechanism is at play. In the next section we will illustrate our results in a more detailed way. For reasons of clarity we will discuss separately our most important results and their implications: the compatibility effect between the hand posture and the line width, the stronger lateralization found with precision grip, and the stronger effects obtained with biological than with not biological hands. Notice, however, that the effects are deeply interwoven since the same phenomenon underlines all effects, i.e. the activation of motor information while observing hands potentially interacting with objects.

\subsection{The compatibility effect}

Our results confirmed with a different methodology that observing a hand in potential interaction with an object activates visuo-motor cueing, even if hand postures were not task-related. We observed an interaction between grip and line in conditions of grip sameness with biological hands (experiments 1 and 2), consisting of a reduction of the leftward bias (pseudoneglect) with precision over power grip and thin lines, and with power over precision grip and thick lines. Again, we found a compatibility effect between hand posture (precision vs. power) and the presented line (thin vs. thick) in grip diversity conditions. Indeed, the subjective midpoint of the line was directed towards the kind of grip most compatible with the kind of line: subjects biased towards the precision grip with thin lines and towards the power grip with thick lines. We referred to the effect observed with thin line as the precision (grip) effect, and to the one observed with thick line as the power (grip) effect. The presence of both effects reveals that participants anticipated a potential action with the object and suggests that effects of motor simulation were involved. Following this point, results observed in conditions of grip sameness (experiments 1 and 2 ), although partially significant, can be discussed in these terms. Indeed, a marginally significant difference was observed between both precision or power grip and thin line: for biological hands and thin line (experiment 1 ) the leftward bias was reduced more with the precision grip than with the power grip; interestingly, for biological hands and thick line (experiment 2 ) the opposite trend was observed, although it was not significant. It is difficult to define whether this shift towards the right for the more compatible kind of grip to favour interaction with the object is due to the fact that all subjects were right handed or due to the fact that they were using the mouse with their right hand. In our opinion it is nonetheless important to consider that the shift was directed towards the most 
relevant hand for the execution of the movement and for acting with the object.

The compatibility effect observed here complements and extends the findings of behavioural literature on cueing effects. Firstly, it shows that the visuo-motor cueing is not limited to a SRC effect. It is possible to establish that the results observed in the present study are not due to an effect of congruency between the target and the response modality, given that no specific motor response that served to remind participants of a power or precision grip was involved in the task (consider that the hand postures adopted when we use the mouse can be considered as palm and poke rather than as clench and pinch postures, according to the distinction proposed by Klatzky et al., 1987 and Buxbaum \& Kalenine, 2010).

Again, it is important to underline that we found a compatibility effect even with a line, which can be considered more as a stylised object than an object per se. The majority of studies on compatibility between precision and power hand postures and differently graspable objects used stimuli belonging to different categories, such as artifacts and natural objects. In those studies probably the long-term experience of interaction with a given object, mediated by the ventral system, plays a role. For example, our long-term visuomotor experience with cherries may strongly influence responses in a SRC study with cherries. The line is a more abstract stimulus compared to objects such as cherries and hammers. Therefore we can speculate that the role of on-line information, mediated by the dorsal system, plays a major role in explaining the effects. This result is interesting because it indicates that the representation of an object is strictly linked to the possibility to interact with it, which in turn is strictly linked to its physical properties (e.g., size). At a theoretical level, the result may suggest that the distinction initially proposed by Milner and Goodale (1995) between two routes to action, the first implying a direct vision-action mapping, mediated by the dorsal system, and the second involving object knowledge, mediated by the ventral system, might be too strong (see, Gallese, Craighero, Fadiga, \& Fogassi, 1999; Rizzolatti \& Matelli, 2003).

Finally, even a static image of a hand without motor preparation evoked motor information, as found in previous neuroimaging and TMS studies (Johnson-Frey et al., 2003; Pierno et al., 2008b; Urgesi et al., 2006). It is thus possible that more than the dynamic hand movement (as claimed by Vainio et al., 2008) causes this effect; the effect must be generated by a combination of the hand posture and the object characteristics.

\subsection{The precision effect}

The second important result that we observed was that in both experiments 1 and 2, and in experiment 4, precision effects were larger than the power effects. At a theoretical level, this is in keeping with studies showing that the precision grip, typically more associated with artefacts than with natural objects, takes longer to be processed than the power grip as it is associated with functional actions (e.g., Anelli et al., 2010; Borghi et al., 2007). Typically, in the behavioural literature results showing longer RTs with hands displaying a precision grip (or alternatively with objects requiring a precision grip) can be interpreted in different ways: in terms of a higher visual complexity of precision compared to power grip or in terms of motor simulation of an action with a more complex motor execution. In the present study, the explanation according to which the precision grip is perceptually more complex seems not plausible, given that in the conditions of grip sameness (experiments 1 and 2 ) we observed a more lateralized pattern of results when two power grip postures were presented compared to when two precision hand postures were displayed, ruling out the possibility of a larger visual complexity of precision stimuli. Following this point, we claim that in conditions of grip sameness the precision grip evoked more skilled, functional actions, to be preferentially performed with the dominant (right) hand, rather than simple grasping actions. This is in line also with results by Vainio et al. (2007), who asked participants to evaluate the accuracy of grips performed with or without objects; the authors found that the fact that a grasping action is object directed is more important for precision than for power grips.

\subsection{The difference between biological and non biological hands}

The third important result was that the hand-cueing effects were stronger for biological than for non biological hands. We found that the bisection bias was less lateralized when the observed hand was not morphologically similar to the participants' hand, i.e. when the fake hand or the robotic hand was presented. Consider that in our study the reduced degree of morphological similarity suggests a reduced capability to perform similar motor programs, as the non-morphologically similar hands were non-biological hands. This result clearly keeps with the Theory of Event Coding (Hommel et al., 2001) and more generally with common coding theories, and it suggests a reduced capability to step into others' shoes when the events we perceive and the events (actions) we are able to produce are dissimilar.

This finding, i.e. the enhancement of the effect with biological stimuli, has interesting implications concerning the role played by attention in our task. In particular, it rules out the possible alternative explanation that the effect is simply due to the fact that attention is directed towards the most salient part of the scene which is contextually related to the target (e.g., Oliva \& Torralba, 2007). Indeed, our results reveal that attention is drawn by very peculiar contexts, which are not simply perceptually salient: rather, they are contexts where an action is possible. Previous results have shown that the presentation of a hand can work as an efficient cue to direct attention. Craighero et al. (2002) have shown that seeing a hand facilitates grasping when the orientation of the observed hand matches the participant's hand orientation required for the grasping movement (see also Vogt et al., 2003). Fischer et al. (2008) found that observing a precision vs. power hand posture induced action shifts towards objects congruent with the presented posture. In line with premotor theories of attention, our results suggest that attention is directed where a potential action goal (congruent object) is selected.

Beyond showing these effect with a novel method, our study, even if behavioural, allows us to speculate on the possible underlying neural mechanisms. Crucially, the fact that the effects are modulated by the similarity between our hand and another's hand suggests that the possible underlying neural basis for this attention shifting is the mirror neuron system, because otherwise the effects should be the same with biological, robotic or fake hands. It remains to be determined whether this effect is due to perceptual similarity or to similarity in motor competence. In other words, our data do not allow us to determine whether we simulate more when we see hands which are perceptually similar to our own hands, or when we see hands which, due to their biomechanical characteristics, can perform movements which are more similar to the movements which are part of our motor repertoire, as common coding theories would predict and as brain imaging and behavioural studies on motor resonance in athletes suggest (Aglioti, Cesari, Romani, \& Urgesi, 2008; Calvo-Merino, Grezes, Glaser, Passingham, \& Haggard, 2006; Pezzulo, Barca, Lamberti Bocconi, \& Borghi, 2010).

\subsection{The method}

Besides the different cognitive processes involved in this task (perceptual, attentional, and motor), the bisection paradigm was 
successfully adapted to investigate high-level visuo-motor cognitive processing such as visuo-motor cueing. Indeed, results were in line and extended previous finding on this issue, as explained above. For this reason we suggest possible developments and applications of the hand-cueing line bisection task. For example, we suggest that the hand-cueing bisection task can be successfully adapted in future neuropsychological studies to study action representation mechanisms in the context of simulation theories (Buxbaum \& Kalenine, 2010). Symbolic cues (e.g., arrows, eye gazes or digits; emotional faces) have often been used with line bisection in studies on neglect syndrome (respectively: Bonato, Priftis, Marenzi, \& Zorzi, 2008; Tamietto et al., 2005). These studies generally share the underlying assumption that cueing effects in line bisection involve top-down rather than bottom-up processes (but for different interpretation see: Chieffi, 1996; Fischer, 1994). We thus suggest that this paradigm can be used to explore functional and manipulative mechanisms in apraxia and optic ataxia. Where apraxia is characterized by an impairment in the manner in which we manipulate objects to perform skilled actions (function impairment), optic ataxia consists of an impairment in grasping objects independently of function (manipulative impairment). Applying this paradigm in neuropsychological contexts could contribute to the understanding of functional and manipulative object processing.

In conclusion, we suggest that the hand-cueing line bisection task can allow us to understand unresolved issues of motor simulation theories. To elaborate, this task could help us understand and clarify whether observing objects evokes functional vs. manipulative information, whether the activation of motor information is modulated by the perspective (first vs. third person), and whether motor mechanisms are triggered by object- vs. action-observation.

\section{Acknowledgements}

This work was supported by the European Community, in FP7 project ROSSI: Emergence of Communication in Robots through Sensorimotor and Social Interaction (Grant Agreement No: 216125), and part of the work was carried on with the support of the Marino Golinelli Foundation (Bologna, Italy). Thanks to: Kate Burke (NUI Galway, Ireland) for revision of the English text; Luisa Girelli and Carlo Toneatto (University of Milano-Bicocca, Italy) for a preliminary version of the computerized bisection task; Erol Sahin (Middle East Technical University, Turkey) for robotic hands images; people of the EMCOlab (www.emco.unibo.it) for helpful comments.

\section{References}

Aglioti, S. M., Cesari, P., Romani, M., \& Urgesi, C. (2008). Action anticipation and motor resonance in elite basketball players. Nature Neuroscience, 11, 1109-1116.

Anelli, F., Nicoletti, R., \& Borghi, A. M. (2010). Categorisation and action: What about object consistence? Acta Psychologica, 133, 203-211.

Barton, J. J., Behrmann, M., \& Black, S. (1998). Ocular search during line bisection: The effects of hemi-neglect and hemianopia. Brain, 121, 1117-1131.

Bazzarin, V., Borghi, A. M., Tessari, A., \& Nicoletti, R. (2007). Is a small apple more like an apple or like a cherry? In S. Vosniadou, D. Kayser, \& A. Protopapas (Eds.), Proceedings of the European cognitive science conference, 2007 (pp. 83-88). London, UK: Taylor and Francis.

Begliomini, C., Caria, A., Grodd, W., \& Castiello, U. (2007). Comparing natural and constrained movements: New insights into the visuomotor control of grasping. PloS One, 10, e1108.

Begliomini, C., Wall, M., Smith, A. T., \& Castiello, U. (2007). Differential cortical activity for precision versus whole-hand visually guided grasping. The European Journal of Neuroscience, 25, 1245-1252.

Bisiach, E., Capitani, E., Colombo, A., \& Spinnler, H. (1976). Halving a horizontal segment: A study on hemisphere-damaged patients with cerebral focal lesions. Schweizer Archiv fur Neurologie, Neurochirurgie und Psychiatrie, 118, 199-206.

Bonato, M., Priftis, K., Marenzi, R., \& Zorzi, M. (2008). Modulation of hemispatial neglect by directional and numerical cues in the line bisection task. Neuropsychologia, 46, 426-433.
Borghi, A. M., \& Cimatti, F. (2010). Embodied cognition and beyond: Acting and sensing the body. Neuropsychologia, 48, 763-773.

Borghi, A. M., \& Riggio, L. (2009). Sentence comprehension and simulation of object temporary, canonical and stable affordances. Brain Research, 1253, 117-128.

Borghi, A. M., Bonfiglioli, C., Lugli, L., Ricciardelli, P., Rubichi, S., \& Nicoletti, R. (2007). Are visual stimuli sufficient to evoke motor information? Studies with hand primes. Neuroscience Letters, 411, 17-21.

Bruzzo, A., Borghi, A. M., \& Ghirlanda, S. (2008). Hand-object interaction in perspective. Neuroscience Letters, 441, 61-65.

Bub, D. N., Masson, M. E. J., \& Cree, G. S. (2008). Evocation of functional and volumetric gestural knowledge by objects and words. Cognition, 106, 27-58.

Buccino, G., Binkofski, F., Fink, G. R., Fadiga, L., Fogassi, L., Gallese, V., et al. (2001). Action observation activates premotor and parietal areas in a somatotopic manner: An fMRI study. European Journal of Neuroscience, 13, 400-404.

Buxbaum, L. J., \& Kalenine, S. (2010). Action knowledge, visuomotor activation and embodiment in the two action systems. Annals of the New York Academy of Sciences, 1191, 201-218.

Caligiore, D., Borghi, A. M., Parisi, D., \& Baldassarre, G. (2010). TRoPICALS: A computational embodied neuroscience model of experiments on compatibility effects. Psychological Review,

Calvo-Merino, B., Grezes, J., Glaser, D. E., Passingham, R. E., \& Haggard, P. (2006). Seeing or doing? Influence of visual and motor familiarity in action observation. Current Biology, 16, 1905-1910.

Castiello, U. (2003). Understanding other people's actions: Intention and attention. Journal of Experimental Psychology: Human Perception and Performance, 29, 416-430.

Castiello, U., Lusher, D., Mari, M., Edwards, M. G., \& Humphreys, G. W. (2002). Observing a human or a robotic hand grasping an object: Differential motor priming effects. In W. Prinz, \& B. Hommel (Eds.), Attention and performance (pp. 314-334). Cambridge, MA: MIT Press.

Chao, L. L., \& Martin, A. (2000). Representation of manipulable man-made objects in the dorsal stream. Neuroimage, 12, 478-494.

Chieffi, S. (1996). Effects of stimulus asymmetry on line bisection. Neurology, 47, 1004-1008.

Chieffi, S. (1999). Influence of perceptual factors on line bisection. Cortex, 4, 523-536.

Costantini, M., Ambrosini, E., Scorolli, C., \& Borghi, A. M. (2011). When objects are close to me: Affordances in the peripersonal space. Psychonomic Bulletin $\mathcal{E}$ Review, 18, 302-308.

Craighero, L., Bello, A., Fadiga, L., \& Rizzolatti, G. (2002). Hand action preparation influences the responses to hand pictures. Neuropsychologia, 40, 492-502.

Craighero, L., Fadiga, L., Rizzolatti, G., \& Umilta', C. A. (1998). Visuomotor priming. Visual Cognition, 5, 109-125.

Craighero, L., Fadiga, L., Rizzolatti, G., \& Umilta, C. (1999). Action for perception: A motor-visual attentional effect. Journal of Experimental Psychology: Human Perception and Performance, 25, 1673-1692.

Creem-Regehr, S. H., \& Lee, J. N. (2005). Neural representations of graspable objects: Are tools special? Cognitive Brain Research, 22, 457-469.

De Hevia, M. D., Girelli, L., \& Vallar, G. (2006). Numbers and space: A cognitive illusion? Experimental Brain Research, 168, 254-264.

Decety, J., \& Grezes, J. (2006). The power of simulation: Imagining one's own and other's behavior. Brain Research, 1079, 4-14.

Edwards, M. G., Humphreys, G. W., \& Castiello, U. (2003). Motor facilitation following action observation: A behavioural study in prehensile action. Brain and Cognition, 53, 495-502.

Ehrsson, H. H, Fagergren, A., Jonsson, T., Westling, G., Johansson, R. S., \& Forssberg, H. (2000). Cortical activity in precision-versus power-grip tasks: An fMRI study. Journal of Neurophysiology, 83, 528-536.

Ehrsson, H. H., Kuhtz-Buschbeck, J. P., \& Forssberg, H. (2002). Brain regions controlling nonsynergistic versus synergistic movement of the digits: A functional magnetic resonance imaging study. The Journal of Neuroscience, 22, 5074-5080.

Ellis, R., \& Tucker, M. (2000). Micro-affordance: The potentiation of components of action by seen objects. British Journal of Psychology, 9, 451-471.

Fagioli, S., Hommel, B., \& Schubotz, R. I. (2007). Intentional control of attention: Action planning primes action-related stimulus dimensions. Psychological Research, 71, 22-29.

Fischer, H. M., Prinz, J., \& Lotz, K. (2008). Grasp cueing shows obligatory attention to action goals. The Quarterly Journal of Experimental Psychology, 61, 860-868.

Fischer, M. H. (1994). Less attention and more perception in the cued line bisection. Brain and Cognition, 25, 24-33.

Fischer, M. H. (2001). Cognition in the line bisection task. Trends in Cognitive Sciences, $5,460-462$.

Gallese, V. (2009). Motor abstraction: A neuroscientific account of how action goals and intentions are mapped and understood. Psychological Research, 73, 486-498.

Gallese, V., Craighero, L., Fadiga, L., \& Fogassi, L. (1999). Perception through action. Psyche, 5, 5-21.

Gazzola, V., Rizzolatti, G., Wicker, B., \& Keysers, C. (2007). The anthropomorphic brain: The mirror neuron system responds to human and robotic actions. Neuroimage, $35,1674-1684$.

Gerlach, C., Law, I., \& Paulson, O. B. (2002). When action turns into words. Activation of motor-based knowledge during categorization of manipulable objects. Journal of Cognitive Neuroscience, 14, 1230-1239.

Girardi, G., Lindemann, O., \& Bekkering, H. (2010). Context effects on the processing of action-relevant object features. Journal of Experimental Psychology: Human Perception and Performance, 36, 330-340.

Grafton, S. T., Fadiga, L., Arbib, M. A., \& Rizzolatti, G. (1997). Premotor cortex activation during observation and naming of familiar tools. Neurolmage, 6, 231-236. 
Grafton, S. T. (2009). Embodied cognition and the simulation of action to understand others. Annals of the New York Academy of Sciences, 1156, 97-117.

Gonzalez, C., Ganel, T., \& Goodale, M. A. (2006). Hemispheric specialization for the visual control of action is independent of handedness. Journal of Neurophysiology, 95, 3496-3501.

Grèzes, J., \& Decety, J. (2001). Functional anatomy of execution, mental simulation, observation, and verb generation of actions: A meta-analysis. Human Brain Mapping, 12, 1-19.

Hari, R., Forss, N., Avikainen, S., Kirveskari, E., Salenius, S., \& Rizzolatti, G. (1998). Activation of human primary motor cortex during action observation: A neuromagnetic study. Proceedings of the National Academy of Sciences of the United States of America, 95, 15061-15065.

Hommel, B., Müsseler, J., Aschersleben, G., \& Prinz, W. (2001). The theory of event coding (TEC): A framework for perception and action planning. The Behavioral and Brain Sciences, 24, 849-878.

Jax, S. A., \& Buxbaum, L. J. (2010). Response interference between functional and structural actions linked to the same familiar object. Cognition, 115, 350-355.

Jeannerod, M. (2001). Neural simulation of action: A unifying mechanism for motor cognition. Neuroimage, 14, S103-S109.

Jewell, G., \& McCourt, M. E. (2000). Pseudoneglect: A review and meta-analysis of performance factors in line bisection tasks. Neuropsychologia, 38, 93-110.

Johnson-Frey, S. H. (2004). The neural bases of complex tool use in humans. Trends in Cognitive Sciences, $8,71-78$.

Johnson-Frey, S. H., Moloof, F. R., Newman-Norlund, R., Farrer, C., Inati, S., \& Grafton, S. T. (2003). Actions or hand-object interactions? Human inferior frontal cortex and action observation. Neuron, 39, 1053-1058.

Kashmere, J. L., \& Kirk, A. (1997). The complex interaction of normal biases in line bisection. Neurology, 49, 887-889.

Kerkhoff, G., \& Bucher, L. (2008). Line bisection as an early method to assess homonymous hemianopia. Cortex, 44, 200-205.

Klatzky, R. L., McCloskey, B., Doherty, S., Pellegrino, J., \& Smith, T. (1987). Knowledge about hand movements and knowledge about objects. Journal of Motor Behavior $19,187-213$.

Martin, A. (2007). The representation of object concepts in the brain. Annual Review of Psychology, 58, 25-45.

Milner, A. D., \& Goodale, M. A. (1995). The visual brain in action. Oxford: Oxford University Press.

Oliva, A., \& Torralba, A. (2007). The role of context in object recognition. Trends in Cognitive Sciences, 11, 520-527.

Pellicano, A., Iani, I., Borghi, A. M., Rubichi, S., \& Nicoletti, R. (2010). Simon-like and functional affordance effects with tools: The effects of object perceptual discrimination and object action state. The Quarterly Journal of Experimental Psychology, 63, 2190-2201.

Pezzulo, G., Barca, L., Lamberti Bocconi, A., \& Borghi, A. M. (2010). When affordances climb into your mind: Advantages of motor simulation in a memory task performed by novice and expert rock climbers. Brain and Cognition, 73, 68-73.

Pierno, A. C, Mari, M., Lusher, D., \& Castiello, U. (2008). Robotic movement elicits visuomotor priming in children with autism. Neuropsychologia, 46, 448-454.

Pierno, A. C., Tubaldi, F., Turella, L., Grossi, P., Barachino, L., Gallo, P., et al. (2008). Neurofunctional modulation of brain regions by the observation of pointing and grasping actions. Cerebral Cortex, 19, 367-374.
Press, C., Bird, G., Flach, R., \& Heyes, C. (2005). Robotic movement elicits automatic imitation. Cognitive Brain Research, 25, 632-640.

Prinz, W. (1997). Perception and action planning. European Journal of Cognitive Psychology, 9, 129-154.

Rizzolatti, G., \& Craighero, L. (2004). The mirror-neuron system. Annual Review of Neuroscience, 27, 169-192.

Rizzolatti, G., \& Matelli, M. (2003). Two different streams form the dorsal visual system: Anatomy and functions. Experimental Brain Research, 153, 146-157.

Rizzolatti, G., Fadiga, L., Matelli, M., Bettinardi, E., Paulesu, D., Perani, D., et al. (1996). Localization of grasp representations in humans by PET: 1 . Observation versus execution. Experimental Brain Research, 111, 246-252.

Schenkenberg, T., \& Bradford, D. C. E. T. (1980). Line bisection and unilatera visual neglect in patients with neurologic impairment. Neurology, 30, 509517.

Sposito, A. V., Bolognini, N., Vallar, G., Posteraro, L., \& Maravita, A. (2010). The spatia encoding of body parts in patients with neglect and neurologically unimpaired participants. Neuropsychologia, 48, 334-340.

Symes, E., Ottoboni, G., Tucker, M., Ellis, R., \& Tessari, A. (2010). When motor attention improves selective attention: The dissociating role of saliency. The Quarterly Journal of Experimental Psychology, 63, 1387-1397.

Symes, E., Tucker, M., Ellis, R., Vainio, L., \& Ottoboni, G. (2008). Grasp preparation improves change detection for congruent objects. Journal of Experimental Psychology: Human Perception and Performance, 34, 854-871.

Tai, Y. F., Scherfler, C., Brooks, D. J., Sawamoto, N., \& Castiello, U. (2004). The human premotor cortex is mirror only for biological actions. Current Biology, 14, 117-120.

Tamietto, M., Latini Corazzini, L., Pia, L., Zettin, M., Gionco, M., \& Geminiani, G. (2005) Effects of emotional face cueing on line bisection in neglect: A single case study. Neurocase, 11, 399-404

Tipper, S. P., Paul, M., \& Hayes, A. (2006). Vision-for-action: The effects of object property discrimination and action state on affordance compatibility effects. Pychonomic Bulletin E' Review, 13, 493-498.

Tucker, M., \& Ellis, R. (1998). On the relations between seen objects and components of potential actions. Journal of Experimental Psychology: Human Perception and Performance, 24, 830-846.

Tucker, M., \& Ellis, R. (2001). The potentiation of grasp types during visual object categorization. Visual Cognition, 8, 769-800.

Tucker, M., \& Ellis, R. (2004). Action priming by briefly presented objects. Acta Psychologica, 116, 185-203.

Turella, L., Pierno, A. C., Tubaldi, F., \& Castiello, U. (2009). Mirror neurons in humans: Consisting or confounding evidence? Brain and Language, 108, 10-21.

Urgesi, C., Moro, V., Candidi, M., \& Aglioti, S. M. (2006). Mapping implied body actions in the human motor system. The Journal of Neuroscience, 26, 7942-7949.

Vainio, L., Symesa, E., Ellisa, R., Tuckera, M., \& Ottoboni, G. (2008). On the relations between action planning, object identification, and motor representations of observed actions and objects. Cognition, 108, 444-465.

Vainio, L., Tucker, M., \& Ellis, R. (2007). Precision and power grip priming by observed grasping. Brain and Cognition, 65, 195-207.

Vogt, S., Taylor, P., \& Hopkins, B. (2003). Visuomotor priming by pictures of hands: Perspective matters. Neuropsychologia, 41, 941-951. 